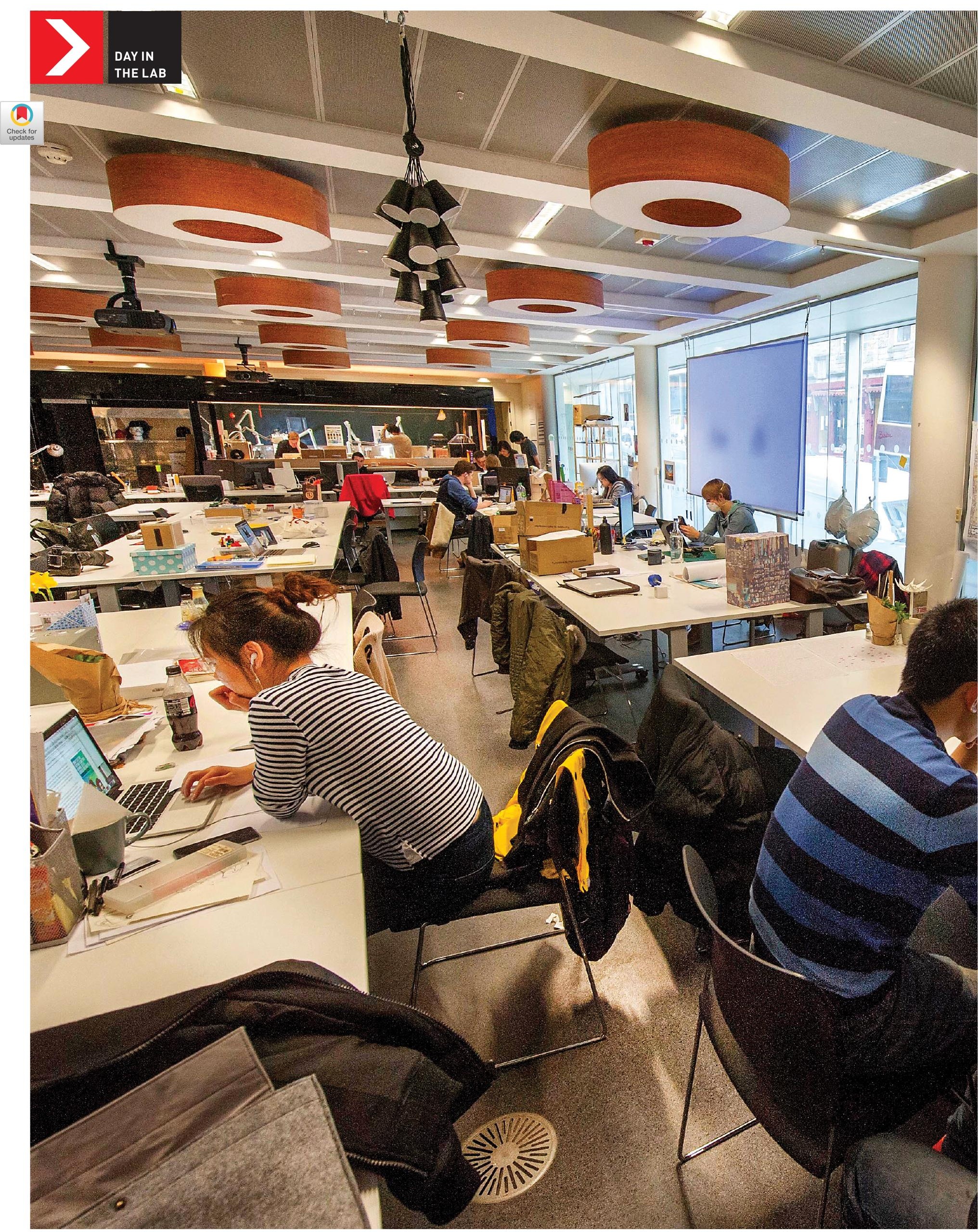




\section{Centre for Design Informatics}

As told by Chris Speed and Jon Oberlander

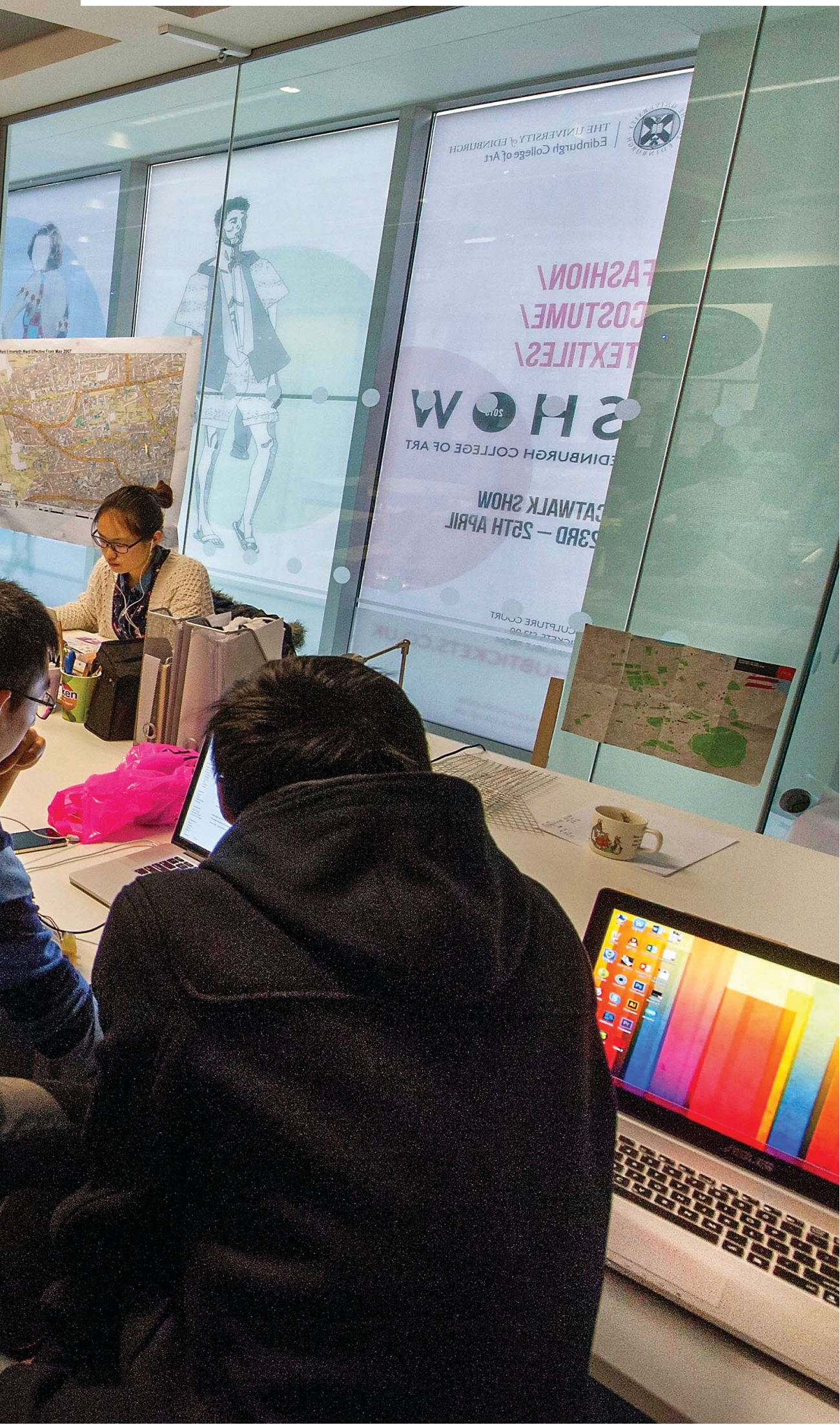

H ow do you describe your lab to visitors? The Centre for Design Informatics provides a platform in which design and data science can mix. As a team, we are interested in the emerging field of human-data interactions and developing ways for design to engage with the complexity of digital economic systems. Through a combination of methods from both the humanities and sciences, the lab offers design-centered solutions for cultural, commercial, and civic sectors, often resulting in new forms of visualizing, experiencing, and interpreting data. The strategic view of Design Informatics is to provide methodologies and solutions that allow organizations to better understand the value of their data in ways that are commensurate with the values of the stakeholders within each network.

What is a unique feature of your lab? The Centre for Design Informatics is situated across the Edinburgh College of Art and the School of Informatics at the University of Edinburgh. Although the footprint of the lab is in the School of Design, we operate in an unusual context across the humanities, science, and engineering, within which a wide variety of lexicons are used to describe the human, the more than human (AI, animal), and the methods for understanding their conditions as they become entangled in different forms of data. In the same way, our materials for exploration are highly mixed and include wood, metal, ceramics, and plastics, as well as machine learning, natural language processing, vision systems, and robotics.

How many people are in the lab, and what is the mix of backgrounds and roles? At the moment we have two professors, Chris Speed and Jon Oberlander, who direct the Centre for Design Informatics. Chris leads on conceptual design and Jon leads on cognitive science and computational linguistics. We have a reader in design informatics (HCI and health informatics) and two lecturers (interaction design and social computing). The lab currently has six active research projects supported by research associates who 
$\rightarrow$ Participants handdrawing map tiles of Edinburgh at the City Selfie Workshop, part of the Edinburgh Art

Festival 2014.
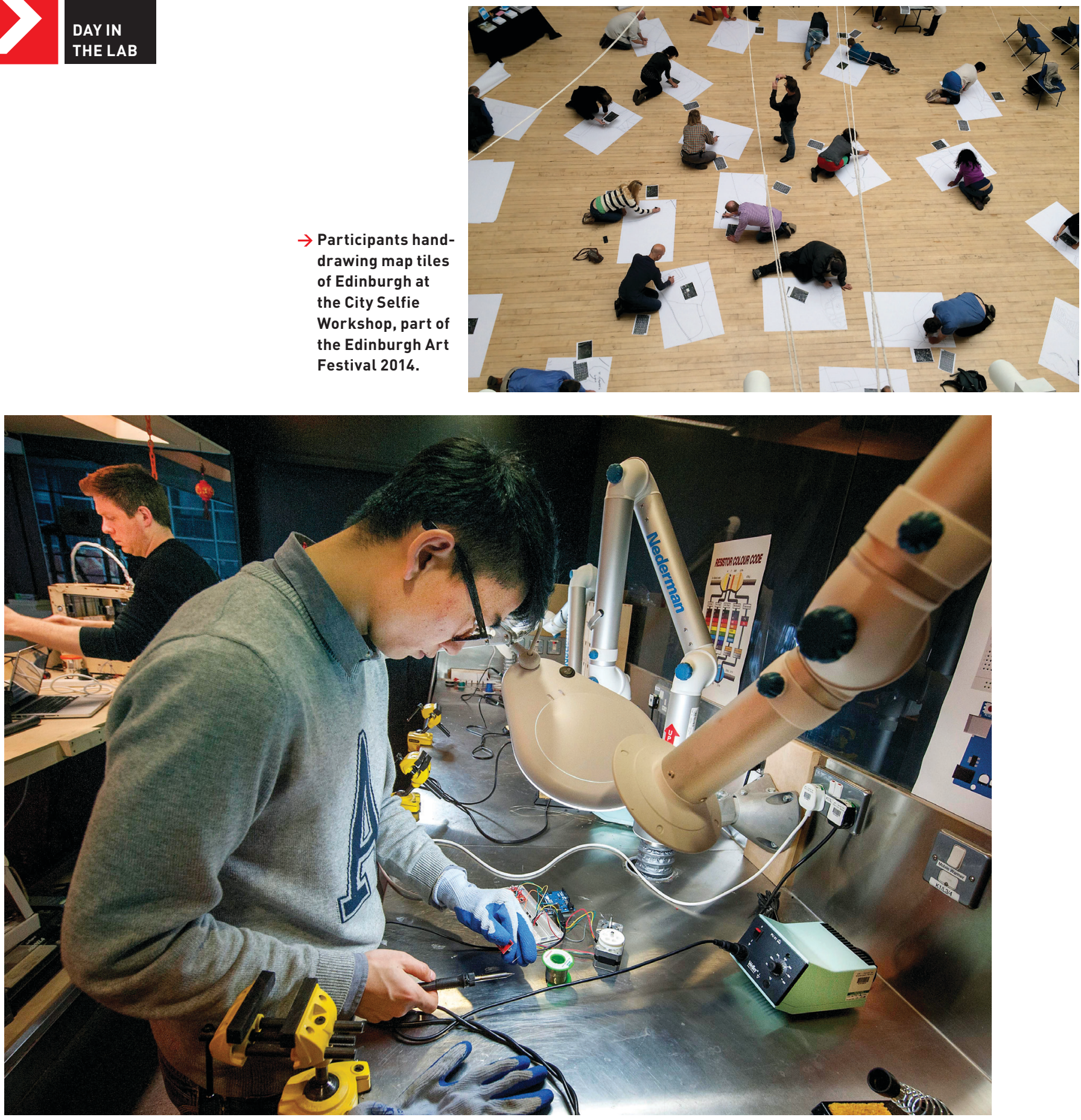

\section{$\rightarrow$ Body paper} prototyping during the Design Meets Synthetic Biology workshop 2016.

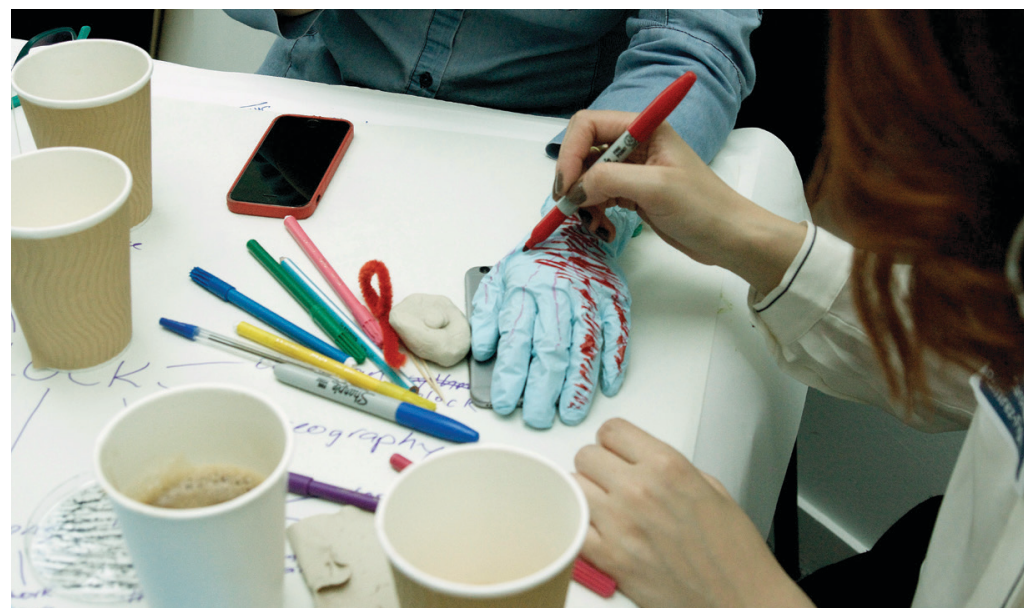




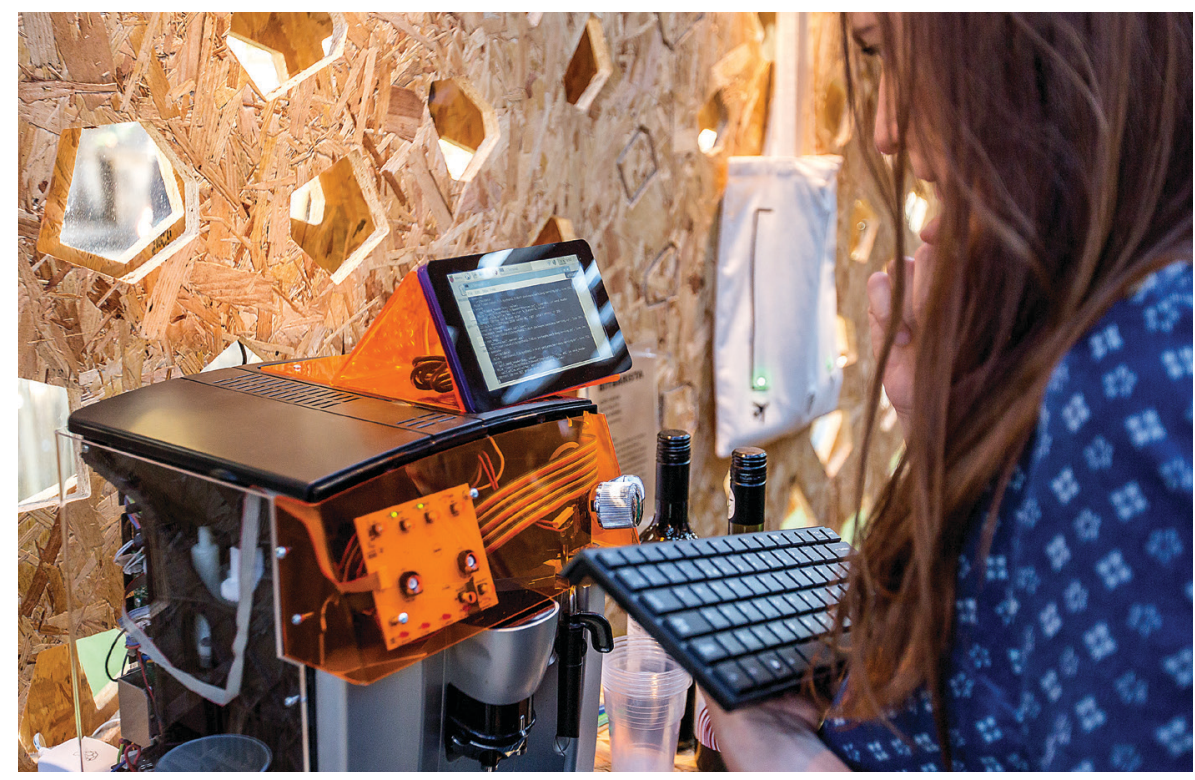

specialize in ethics, consent, and privacy; neuropolitics; anthropology; user experience; human geography; and digital cultures, underpinned by a designer and two software engineers who help bring everything together into meaningful experiences. The research lab is also the heart of M.A. and M.Sc. programs in design informatics, and a Ph.D. community of designers and computer scientists.

\section{Briefly describe a day in the life of} your lab. The highly interdisciplinary team collaborates across research projects that involve organizations such as international charities, banks, museums, and galleries. On any given day, the team will be trying to understand the social, economic, and environmental implications for the flow of data between humans, machines, and designed artifacts. More than likely you will find the team huddled around a device such as the BitBarista, a hacked domestic coffee machine that only accepts Bitcoin and that insists on asking coffee drinkers where it should buy its next bag of coffee. Someone will be trying to understand the environmental and social impact of the material and digital economies of the coffee machine, while someone else will be hacking the software to allow a designer to provide an interface that makes the user aware of the data-value chains involved in ordering a cup of coffee.

\section{What is one feature of your lab that} you could not do without? The mix of people. All experts in their own fields, they offer different insights into every action that we make and offer new tactics when we get stuck with a particular material or method. Sometimes you'll find a designer and software engineers projecting into a near future, but you need the anthropologist and the human geographer to offer pause for reflection to calibrate ethical issues. On other occasions, projects slow down due to complex social and philosophical discussions, and the imagination of the designers allows us to turn ideas into experiences to better understand an issue. We need the mix of people to develop the criticalities around action and reflection.

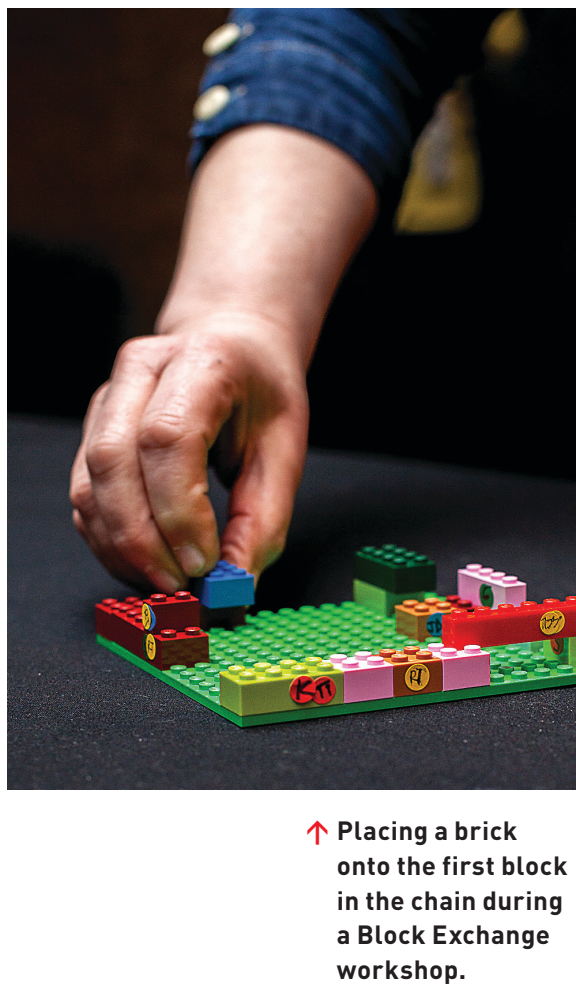

$\leftarrow$ Last minute updates to the BitBarista, a Bitcoin coffee machine, at the Edinburgh Digital Entertainment Festival 2016.

What is one feature of your lab you want and do not have? Children, older people, and animals. As a relatively young department in a large university, we find ourselves surrounded by people of the same age range and genetic makeup. We have the city of Edinburgh and the many participants in the projects to keep us grounded, and we are part of a Living Lab project with the Edinburgh City Council. But the chance to have different perspectives on being human and more than human in the lab would certainly lead to more diverse outcomes.

\section{What is the one thing you see as} most important about the work you do there? Given the complex digital entanglements in which we find ourselves, we consider the exploration of human-centered approaches to data interactions to be of great importance. By developing ways to design from, with, and by data, we think that an interdisciplinary approach can bring data science into the design studio and into design research.

http://www.designinformatics.org 